\title{
Efficacy of Phage Therapy in Controlling Rabbit Colibacillosis and Changes in Cecal Microbiota
}

\author{
Jian Zhao ${ }^{1,2}$, Yan Liu', Chenwen Xiao'2, Shaojie $\mathrm{He}^{2}$, Huochun $\mathrm{Yao}^{1}$ and Guolian Bao ${ }^{2 *}$ \\ ${ }^{1}$ College of Veterinary Medicine, Nanjing Agricultural University, Nanjing, China, ${ }^{2}$ Institute of Animal Husbandry and \\ Veterinary Science, Zhejiang Academy of Agricultural Sciences, Hangzhou, China
}

OPEN ACCESS

Edited by:

Heather K. Allen,

National Animal Disease Center,

United States

Reviewed by:

Maia Merabishvili,

Queen Astrid Military Hospital,

Belgium

Elizabeth Martin Kutter,

The Evergreen State College,

United States

*Correspondence:

Guolian Bao

baoguolian@163.com

Specialty section:

This article was submitted to

Antimicrobials, Resistance

and Chemotherapy,

a section of the journal

Frontiers in Microbiology

Received: 08 March 2017

Accepted: 12 May 2017

Published: 29 May 2017

Citation:

Zhao J, Liu Y, Xiao C, He S, Yao H and Bao G (2017) Efficacy of Phage

Therapy in Controlling Rabbit

Colibacillosis and Changes in Cecal Microbiota. Front. Microbiol. 8:957. doi: 10.3389/fmicb.2017.00957
Phage therapy is a valid weapon that we could use to fight against pathogens. Bacteriophages kill bacteria and self-proliferate in the digestive tract. Furthermore, it was assumed that phage therapy could preserve the existing gut microbiota. In this study, 45 rabbits were equally divided into three groups after they were orally inoculated with pathogenic Escherichia coli to induce gut infection. Each group was treated with bacteriophage ZRP1 (Group P), ciprofloxacin lactate (Group A), or phosphate-buffered solution (PBS) (Group N). Another 15 healthy rabbits composed the control group (Group C). The body weight gain decreased significantly, but the white blood cell (WBC) count, especially the percentage of large WBCs, and the serum endotoxin levels increased significantly after infection. The result of microscopic examination of the ileum showed that E. coli ZR1 adhered to villi and caused hemorrhage inside the villi. Groups $\mathrm{P}$ and $\mathrm{A}$ rabbits recovered after treatments, and both bacteriophage and antibiotic treatment significantly decreased the eaeA gene concentration in cecal contents. The microbiota in cecal contents changed in infected rabbits that were treated with PBS. The relative abundance of Clostridiales and YS2 decreased but the relative abundance of Enterobacteriales increased significantly. According to the principal components analysis, the microbiota of Groups $\mathrm{P}$ and $\mathrm{C}$ rabbits were similar to one another in type and relative abundance but different from those of Groups $\mathrm{N}$ and $\mathrm{A}$ rabbits. The results demonstrated that oral administration of bacteriophage can cure gut infection with minimal impact on the cecal microbiota.

Keywords: bacteriophage, phage therapy, rabbit, colibacillosis, microbiota

\section{INTRODUCTION}

Colibacillosis, caused by pathogenic Escherichia coli and characterized by diarrhea or systematic infection, is a common bacteriosis and generates huge economic losses in the animal husbandry industry. In the last two decades, researchers have investigated alternative treatment methods for colibacillosis because of the antibiotics residues and the increasing concern over drugresistant bacterial strains. Among the promising new agents are bacteriophages, which can specifically lyse host bacterial cells and reproduce themselves approximately one 100-fold, thereby preventing and controlling the disease. Several studies demonstrated that a single orally administrated bacteriophage or bacteriophage cocktail reduced the levels of pathogenic bacteria in the gastrointestinal tract of sheep or mice experimentally infected with E. coli (Tanji et al., 2005; Raya et al., 2011) and decreased the rate of diarrhea and the mortality of poultry in farms (Xie et al., 2005). 
Rabbits have suffered from colibacillosis caused by enteropathogenic E. coli (EPEC) since the beginning of the 1980s (Milon et al., 1999). We recently isolated an atypical EPEC strain (ZR1) and one of its specific bacteriophages (ZRP1) from rabbits. ZRP1 is a member of myoviridae family and its genome is a 68201-bp dsDNA molecule with a GC content of $46.16 \%$. The result of blast showed that the DNA shared $96 \%$ of identity with the Escherichia phage ECML-117 (Query coverage $=93 \%$; $E$-value $=0.0$ ), which is a lytic bacteriophage with a broad target range (Abuladze et al., 2008) and was proved free of bacterial toxin genes, antibiotic resistance encoding genes, and bacterial 16S rRNA genes (Carter et al., 2012). An in vivo trial with ZRP1 as an intravenous therapeutic phage showed that it can significantly prolong the survival time of rabbits simultaneously received lethal dose of ZR1 (Zhao et al., 2017). Furthermore, we conducted a trial in rabbits to compare the efficacy of orally administered bacteriophage with antimicrobials and to examine the effect of this phage therapy on the intestinal microbiota profiles.

\section{MATERIALS AND METHODS}

\section{Animals Management and Experimental Design}

Rabbits in the experiment were raised in cages under controlled temperature $\left(20 \pm 2^{\circ} \mathrm{C}\right)$ and natural light conditions. Animals had access to water ad libitum and were fed $80 \mathrm{~g}$ of feed per day per head. The diet mainly comprised hay powder and bran, which provided $15.8 \mathrm{MJ} / \mathrm{kg}$ of general energy, $14 \%$ crude protein, and $18 \%$ crude fiber. The rabbits also received vitamins and trace minerals consistent with the nutrient requirements for growing rabbits. Antibiotics were removed from their diets but anticoccidial drugs were kept.

The study was divided into two stages. In the first stage, 60 New Zealand white rabbits (non-specific pathogen free [SPF]) with an average body weight of approximately $1 \mathrm{~kg}$ were allocated to two groups according to their body weights and white blood cell (WBC) counts. The control group (Group C) comprised 15 rabbits free of experimental infection with E. coli. Group B was composed of 45 rabbits that were orally inoculated with $1 \mathrm{ml}\left(1 \times 10^{10} \mathrm{CFU} / \mathrm{ml}\right)$ of E. coli ZR1. In the second stage of the experiment, Group B rabbits were weighed, and their blood samples were collected for WBC count at 3 days post-infection. Based on the body weights and WBC counts, the 45 rabbits were equally divided into three groups: negative control group (Group $\mathrm{N}$ ), phage-therapy group (Group P), and antibiotic-therapy group (Group A). After grouping, rabbits were immediately orally treated with $1 \mathrm{ml}$ PBS, $1 \mathrm{ml}$ bacteriophage ZRP1 $\left(1 \times 10^{11} \mathrm{PFU} / \mathrm{ml}\right)$, and $1 \mathrm{ml}$ ciprofloxacin lactate $(20 \mathrm{mg} / \mathrm{ml})$, respectively. Group C rabbits received $1 \mathrm{ml}$ PBS orally each time in order to create the same physical stress in those rabbits. Three days after treatments, all rabbits were weighed, bled for blood samples, and killed for cecal contents collection.

\section{Histology of lleum after E. coli Experimental Infection}

Another two rabbits were sacrificed 3 days after oral infection with E. coli in order to investigate whether ZR1 could adhere to the villi of the small intestine. Formalin-fixed ileum specimens were embedded and cut into sections. Sections were stained with hematoxylin and eosin and viewed under an Olympus BX51 light microscope (Olympus [China] Co., Ltd, China).

\section{Analyses of Blood Samples}

Blood samples were taken from the ear vein by pyrogenfree syringes at 1 day pre-infection, 3 days post-infection, and 3 days post-treatment (6 days post-infection). Each time, $40 \mu \mathrm{l}$ of fresh blood were mixed with heparin sodium; and the WBC counts, as well as the rates of large white blood cells (W-LCR), were examined by an automatic hematology analyzer (pocH-100iV Diff, SYSMEX Shanghai Ltd, China).

Approximately $2 \mathrm{ml}$ of coagulated blood from each rabbit were centrifuged $(8,000 \mathrm{~g} ; 10 \mathrm{~min})$ for serum in pyrogen-free polyethylene tubes. The lipopolysaccharide (LPS) concentrations were determined by a quantitative chromogenic end-point tachypleus amebocyte lysate (TAL) kit (Chinese Horseshoe Crab Reagent Manufactory Co., Ltd, China) according to the manufacturer's instruction. Briefly, $100 \mu \mathrm{l}$ samples were incubated with $100 \mu \mathrm{l}$ of TAL solutions in non-pyrogen tubes at $37^{\circ} \mathrm{C}$ for $15 \mathrm{~min}$; then, $100 \mu \mathrm{l}$ pre-warmed synthetic substrates were added and followed by another 6 min of incubation. Finally, diazo reagents were added; and the absorbance at $545 \mathrm{~nm}$ was measured after standing for $5 \mathrm{~min}$. The LPS concentrations were calculated by the standard curve method (Wei et al., 2015).

\section{Quantitative Real-Time PCR (qRT-PCR) of eaeA}

DNA from $0.2 \mathrm{~g}$ of cecal contents from each sample was extracted using a QIAamp ${ }^{\circledR}$ fast DNA stool mini kit (QIAGEN ${ }^{\circledR}$ Co., Ltd, Germany) according to the manufacturer's instructions.

The target sequence quantified was a fragment (384 bp) of eaeA gene from E. coli ZR1. The primers used for the amplification were F-5' GAC CCG GCA CAA GCA TAA GC $3^{\prime}$ and R-5' CCA CCT GCA GCA ACA AGA GG 3' (Luz Maria Chacon et al., 2012). The qRT-PCR was performed on an ABI 7300 system (Applied Biosystems, Thermofisher Scientific Inc., United States). Each experiment was carried out in duplicate with a final volume in each well of $25 \mu$ l, which consisted of $12.5 \mu \mathrm{l}$ SYBR $^{\circledR}$ Premix Ex Taq (Takara Biotechnology [Dalian] Co., Ltd, China), $5 \mu$ l DNA samples, $0.5 \mu$ l Rox Reference Dye (Takara Biotechnology [Dalian] Co., Ltd, China), $0.5 \mu l$ of each primer, and $6 \mu \mathrm{ldd} \mathrm{H}_{2} \mathrm{O}$. The program was set following the software wizard (software version 1.4), and 40 cycles were run alternately at $95^{\circ} \mathrm{C}$ for $5 \mathrm{~s}$ and $60^{\circ} \mathrm{C}$ for $30 \mathrm{~s}$. The specificity of the reaction was checked using a dissociation curve. Pure eaeA fragments of known concentration were diluted 10 -fold serially to create the standard curve, and the eaeA concentration of each sample 
was calculated by interpolating the $C_{t}$ value to the standard curve.

\section{Analysis of Cecal Microbiota}

In each group, three to four DNA samples (see Quantitative RealTime PCR [qRT-PCR] of eaeA) were pooled so that four mixed samples were used for amplifications and the HiSeq sequencing. Universal primers 341F (5' CCT ACG GGN GGC WGC AG $3^{\prime}$ ) and $805 \mathrm{R}$ ( $5^{\prime}$ GAC TAC HVG GGT ATC TAA TCC $3^{\prime}$ ) were used to amplify fragments that covered the $\mathrm{V} 3$ and $\mathrm{V} 4$ regions of the 16S rRNA genes (Vasileiadis et al., 2012). A HiSeq 2500 system (Illumina Inc., United States) was utilized for sequencing using the PE250 run type. Clean reads were assembled, and aligned tags were clustered at a $97 \%$ similarity level into operational taxonomic units (OTUs) referencing the Greengenes 16S rRNA gene database. Microbiota profiles were examined by QIIME for Venn diagram (Bauerl et al., 2014) and $\mathrm{R}$ (version 3.3.3) for principal components analysis (PCA) (Combes et al., 2014).

\section{Statistical Analyses}

The statistical software SPSS 17.0 was used to analyze all data except those of microbiota profiles. Least significant difference in one-way analysis of variance was used for multiple comparisons among groups. Square root transformation and log transformation were adopted to render the percentage and qRT-PCR data subject to normal distribution. Additionally, the Kruskal-Wallis $H$ test was used to separate differences in bacterial relative abundance among groups.

\section{Ethics}

The study was performed according to the National Institutes of Health Guidelines for the Care and Use of Laboratory Animals (NIH Publication No. 85-23, revised 1996) and was approved by the Animal Care and Use Committee of Institute of Animal Husbandry and Veterinary Science, Zhejiang Academy of Agricultural Sciences.

\section{RESULTS}

\section{Adhesion of E. coli ZR1 to the lleal Epithelia}

Intestinal sections from the two rabbits used to investigate ZR1 adherence demonstrated colonization of the ZR1 on villi (Figures 1A,B). The integrity of the epithelia was indistinct, and debris attached to E. coli was observed, which indicated inflammation and hemorrhage.

\section{Status of Rabbits in Stage 1}

Slight reduction of body weight was observed in the infected group. However, these rabbits exhibited only half of the body weight gain than what was observed in the healthy animals $(P<0.05)$. A significant increase of blood leukocytes, especially neutrophils, occurred after infection in Group B compared to the control group $(P<0.05)$. The endotoxin concentration in the infected rabbits doubled post-infection $(P<0.05)$. Data mentioned above are presented in Table 1.

\section{Status of Rabbits in Stage 2}

Forty-five infected rabbits were equally divided into three homogeneous groups with very similar weight, WBC or W-LCR values, and serum LPS concentrations. After the treatments, rabbits that received antibiotics had the highest weight gain among all groups $(P<0.05)$ though their average weight was less than that of the control group because of weight-gain lag in the first stage. Rabbits orally inoculated with bacteriophage ZRP1 gained $10 \mathrm{~g}$ more than the gain of those without treatment, but the difference was not statistically significant. The WBC number kept increasing except in Group A, but the larger WBCs and the serum endotoxin units decreased at different rates in all groups. The data showed that the leukocyte types and the LPS values of rabbits treated with either the bacteriophage or the antimicrobial returned to normal levels. Additionally, no significant difference in the WBC number was found between groups A and C. Related data are shown in Table 2.
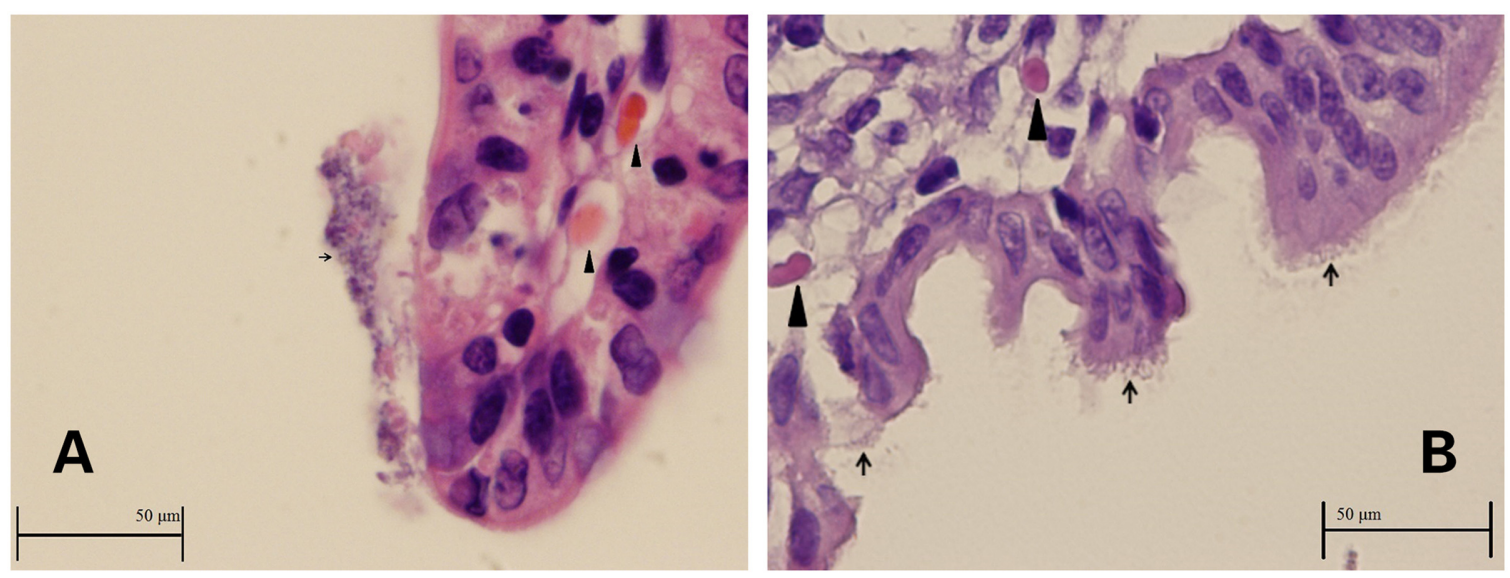

FIGURE 1 | (A,B) Images of ileum from rabbits infected with ZR1. Enterocytes and debris covered with Escherichia coli (thin arrows) and hemorrhage inside the villus (thick arrowheads). 
TABLE 1 | Body weight, body weight gain, WBC count, W-LCR, and serum LPS concentration of rabbits in different groups before and after Escherichia coli infection (Mean $\pm \mathrm{SD}$ ).

\begin{tabular}{lcc}
\hline & Group C & Group B \\
\hline Initial body weight $(\mathrm{kg})$ & $0.972 \pm 0.239$ & $0.975 \pm 0.192$ \\
Body weight after infection $(\mathrm{kg})$ & $1.111 \pm 0.236$ & $1.054 \pm 0.185$ \\
Body weight gain 1 $(\mathrm{kg})$ & $0.139 \pm 0.016^{\mathrm{a}}$ & $0.079 \pm 0.084^{\mathrm{b}}$ \\
Initial WBC count $\left(10^{3} / \mu \mathrm{L}\right)$ & $4.3 \pm 1.4$ & $4.3 \pm 1.2$ \\
WBC count after infection $\left(10^{3} / \mu \mathrm{L}\right)$ & $4.4 \pm 1.6^{\mathrm{a}}$ & $6.5 \pm 2.0^{\mathrm{b}}$ \\
Initial W-LCR $(\%)$ & $12.2 \pm 2.8$ & $11.8 \pm 3.4$ \\
W-LCR after infection $(\%)$ & $9.3 \pm 2.6^{\mathrm{a}}$ & $16.4 \pm 7.8^{\mathrm{b}}$ \\
Initial Serum LPS $(\mathrm{EU} / \mathrm{ml})$ & $0.21 \pm 0.05$ & $0.22 \pm 0.06$ \\
Serum LPS after infection $(\mathrm{EU} / \mathrm{ml})$ & $0.26 \pm 0.09^{\mathrm{a}}$ & $0.50 \pm 0.09^{\mathrm{b}}$ \\
\hline
\end{tabular}

Means with no common superscript letter differ significantly $(P<0.05)$.

\section{Estimation of ZR1 Amount in the Cecal Residues}

Quantitative RT-PCR was performed to estimate the presence of ZR1 by the specific eaeA gene and to confirm differences in amount among groups. The $R^{2}$ of the standard curve established in this study reached 0.94 . The statistical test showed that the eaeA gene level of Group N rabbits was significantly higher than in the others $(P<0.05)$. Accordingly, the numbers of tags representing Enterobacteriaceae demonstrated the same trend (Table 3). A total of 27 OTU types at family Enterobacteriaceae level were clustered; and the dominant one was OTU 782953, which is annotated as Escherichia/Shigella at the genus level. Group N occupied 26 types in the 27 OTUs, while Groups C, P, and A had 3, 6, and 3 types, respectively.

\section{Cecal Bacterial Populations of Different Groups}

A total of 1,274 OTUs were obtained from all samples (Supplementary Table S1). They belonged to 42 orders in 34 classes in 15 phyla. Approximately $50 \%$ of the OTUs cannot be identified at the family level, but almost $99.99 \%$ of OTUs were clear at the order level. Thus, the data were analyzed and shown at the order level to minimize loss of information.

In the Venn diagram (Figure 2), all groups shared 14 orders in common, which accounted for more than $99 \%$ of the relative abundance. Another eight common orders were shared by all infected groups. Group P possessed 39 orders including 13 orders in particular, while Groups C, N, and A possessed 19, 28, and 24 orders, respectively.

The relative abundance of Enterobacteriales in Group $\mathrm{N}$ increased significantly; however, those of the Clostridiales and YS2 significantly decreased. No considerable differences were found among the other three groups. The orders with relative abundance higher than $0.1 \%$ in each group are listed in Table 4.

A PCA was carried out on 16 taxonomic profiles and tended to separate all samples into two clusters as demonstrated on the PC1-PC2 plot, in which PC1 and PC2 explained 52.04 and $14.82 \%$ of the total variation. One cluster comprised Groups $\mathrm{C}$ and $\mathrm{P}$, and the other cluster consisted of Groups $\mathrm{N}$ and $\mathrm{A}$ (Figure 3). The main differences in PC2 values indicate that the cecal microorganisms of the two clusters were compositionally distinct from each other in the second principal component. The PC2 was positively correlated to Burkholderiales but negatively correlated to RF32 and YS2. That is to say, the high level of Burkholderiales and the low level of RF39 and YS2 of Group N or Group A raised their scores in the PC2 axis. Furthermore, the slight difference in PC1 value between the two clusters was mainly caused by the variations of Clostridiales (positive) and Bacteroidales (negative). The PC3 which explained $6.97 \%$ of the total variation showed a negative correlation with Enterobacteriales. So the significantly high percentage of Enterobacteriales in Group N lowered its value in PC 3 axis (Figure 4).

\section{DISCUSSION}

Atypical EPEC causes intestinal lesions characterized by attaching and effacing $(\mathrm{A} / \mathrm{E})$, and the intimin encoded by the attaching and effacing gene (eae) is thought to contribute to the attachment and destruction of microvilli (Hernandes et al., 2009). In our study, histopathological features in the ileum were similar to those previously described (Al-Mamun et al., 2013). Normal intestinal barriers are breached by pathogenic E. coli colonization; then bacteria and their products, such as LPS, infiltrate into blood and recruit polymorphonuclear (PMN) leukocytes. Moreover, as reported previously (Gallois et al., 2007; Al-Mamun et al., 2013),

TABLE 2 | Body weight, body weight gain, WBC count, W-LCR and serum LPS concentration of rabbits with different treatments (Mean \pm SD).

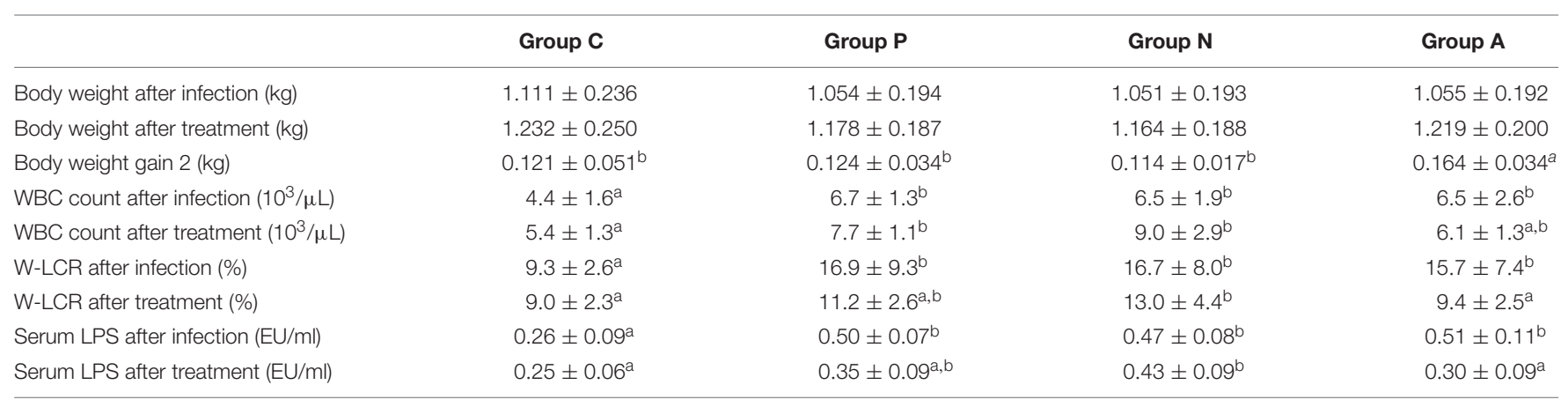

Means with no common superscript letter differ significantly $(P<0.05)$. 
TABLE 3 | eaeA gene levels and sums of Enterobacteriaceae tags in rabbit cecal contents (Mean \pm SD or Sum).

\begin{tabular}{|c|c|c|c|c|}
\hline & Group C & Group P & Group N & Group A \\
\hline eaeA gene concentration $\left(10^{-6} \mu \mathrm{g} / \mu \mathrm{L}\right)$ & $0.16 \pm 0.22^{a}$ & $1.07 \pm 0.78^{a}$ & $16.03 \pm 26.58^{b}$ & $0.14 \pm 0.07^{a}$ \\
\hline Number of Enterobacteriaceae tags & $17^{\mathrm{a}}$ & $74^{\mathrm{a}}$ & $7265^{b}$ & $3^{a}$ \\
\hline
\end{tabular}

Means with no common superscript letter differ significantly $(P<0.05)$.

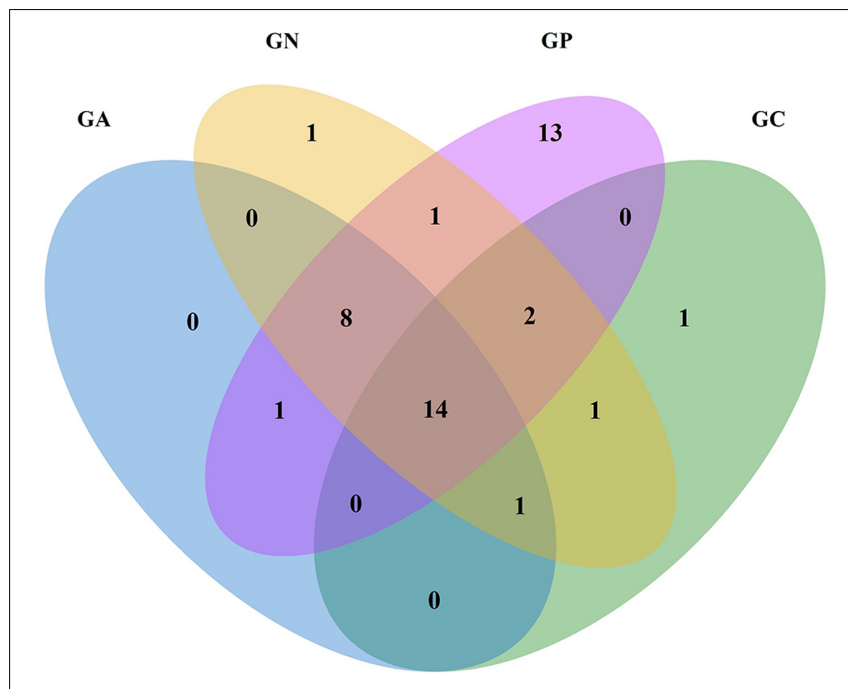

FIGURE 2 | Venn diagram of four groups at the order level according to the classification of the bacterial 16S rRNA V3 and V4 regions. GC, control group; GP, phage-therapy group; GN, negative control group; GA, antibiotic-therapy group.

growth performances of rabbits are retarded by the infection. And, in our case, the retardation was caused by the decrease in the feed conversion ratio as the feed supply was constant. LPS induces an inflammatory response from animal immune systems by binding to toll-like receptor 4 . Intraperitoneal injection of E. coli LPS has been used to stimulate an inflammatory state and trigger the increase in WBCs 24 or $48 \mathrm{~h}$ after the injection (Brecchia et al., 2010, 2014). In our study, the elevation of blood endotoxin levels and the increase in WBC number were also observed in infected rabbits. In additional, the rise in W-LCR indicated that neutrophils responded quickly to the infection during the acute phase of inflammation.

Phage therapy predates the discovery of penicillin and has been investigated as an alternative to antibiotics recently. Bacteriophages are harmless to animals, and their efficacy is superior to that of antibiotics in certain respects (Smith and Huggins, 1982; Bull et al., 2002; Zhang et al., 2015). In our experiment, application of ZRP1 successfully reduced the E. coli ZR1 load in the intestine. Either the eaeA gene abundance or the Enterobacteriaceae tag numbers were substantially lower in treated as compared to in untreated rabbits. qRT-PCR is more sensitive and specific than conventional culture method for diagnosis and quantification (Bischoff et al., 2005). Therefore, the eae gene was selected to identify certain EPEC strain in feces using this method (Al-Mamun et al., 2013). Theoretically, the $e a e A$ gene should not have been detected in the control group.
One possible reason we detected in our control group is because the rabbits we used in the experiment were non-SPF animals and might have carried very few bacteria with the eae gene prior to the start of the experiments. With the elimination of ZR1 in the gastrointestinal tract, serum LPS concentrations and the W-LCR values decreased accordingly, hence the body weight gain recovered after the phage treatment. Ciprofloxacin lactate was more powerful than we expected in treating ZR1 in vivo. This treatment nearly wiped out the pathogenic E. coli; and a consequent compensatory growth was observed in the rabbits. We assumed that the inferior phage therapy efficacy could be the results of using an insufficient phage dose. The influence of bacteriophage-to-bacterium ratio was reviewed (Hraiech et al., 2015); and a previous study concluded that a larger phage dose was superior in passive treatments (Tsonos et al., 2014). The sensitivity of ZR1 to ciprofloxacin lactate may play a key role as well. The studies we cited above (Smith and Huggins, 1982; Bull et al., 2002; Zhang et al., 2015) did not mention the relationships between the pathogens and the antibiotics, but we chose ciprofloxacin lactate by antimicrobial susceptibility testing. Nevertheless, bacteriophages demonstrate a virtue not apparent in antibiotics: phages self-replicate rather than being metabolized in vivo. Thus, dosing bacteriophages repeatedly is unnecessary (Mandal et al., 2014). In our research, numerous active bacteriophages ( $>10^{5} \mathrm{PFU} / \mathrm{g}$ feces, data not shown) were found 3 days after the treatment. Taken the coprophagy of rabbits into account, these bacteriophages had a great chance to re-enter the digestive tract. Moreover, these bacteriophages might play a role in limiting host $E$. coli numbers in the environmental reservoir. Thus, the potential benefits of phage therapy may be seen long term.

After birth, rabbits are colonized by a complex and dynamic consortium of microorganisms in the digestive tract; and the bacterial community alteration could be caused by inflammation. In our study, the Venn diagram shows that Groups P, N, and A had eight orders in common. This may be attributed to the altered intestinal environment caused by the experimental infection of E. coli ZR1, creating niches for those bacteria to grow. However, the emergence of 13 particular orders in cecal contents of phagetherapy rabbits is still a mystery.

Using a highly effective sequencing technique, the representation of some cecal bacteria was established. Except Cyanobacteria, four phyla (Firmicutes, Bacteroidetes, Tenericutes, and Verrucomicrobia) in the top five and their relative abundances in the cecum of healthy rabbits were very similar to those previously reported (Bauerl et al., 2014). Likely, in rabbits infected but untreated, the increase of Enterobacteriales, Bacteroidales, and Verrucomicrobiales accompanied by a decrease of Clostridiales were observed, 
TABLE 4 | Influence of different treatments on the bacterial core order relative abundance (\%) in all groups (Mean \pm SD).

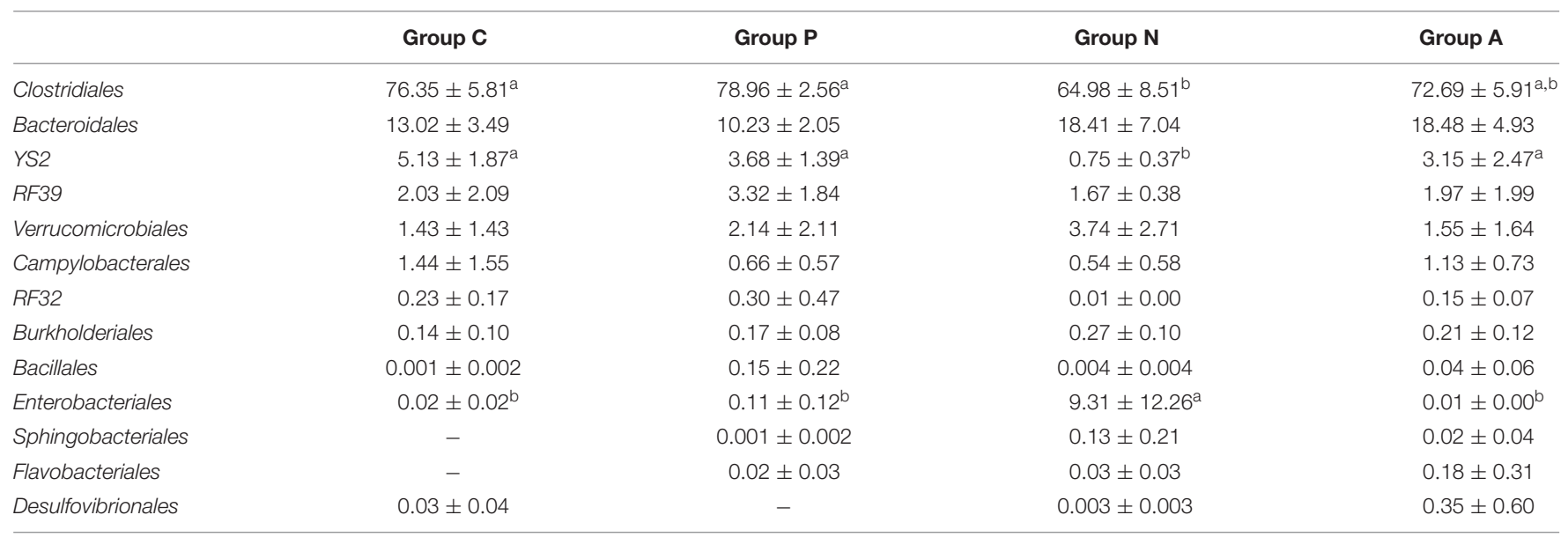

Means with no common superscript letter differ significantly $(P<0.05)$.

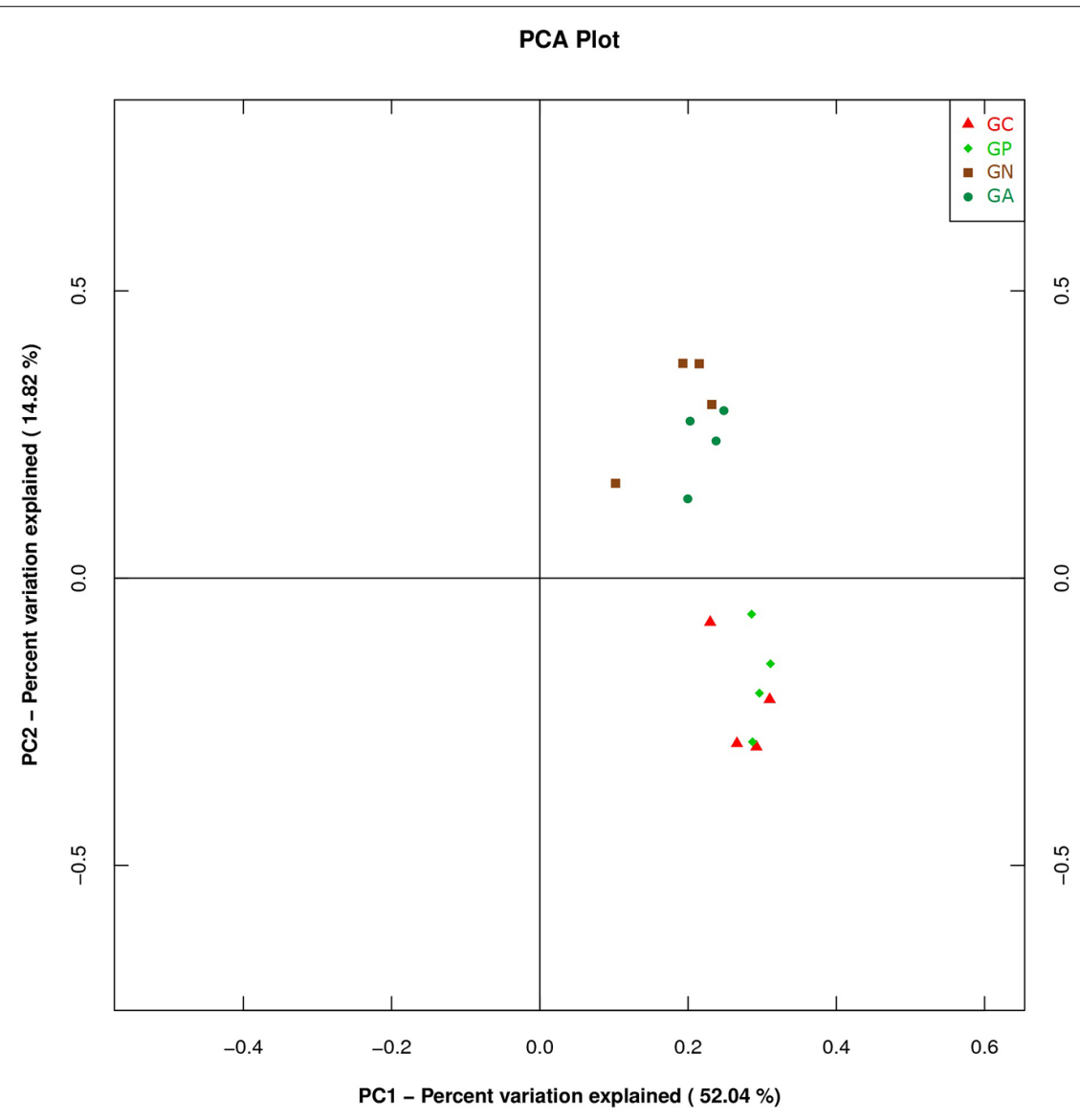

FIGURE 3 | Principal component analysis (PCA) plot based on the first and second PC of bacterial community taxonomic profiles from 16 mixed cecal samples in four groups. GC, control group; GP, phage-therapy group; GN, negative control group N; GA, antibiotic-therapy Group.

too (Bauerl et al., 2014). The results indicated a negative correlation between Clostridiales and the pathogens when the intestines of healthy rabbit were infected. Nevertheless, Gut bacteria plays an important role in food fermentation and therefore in health status. The rabbit's cecal microbial composition was dominated by Ruminococcaceae (in the order Clostridiales) which succeeded Bacteroidaceae (in the order Bacteroidales) after weaning to adapt solid feed and such alteration reduced the mortality of weaned kits (Combes et al., 2014). 


\section{PCA Plot}

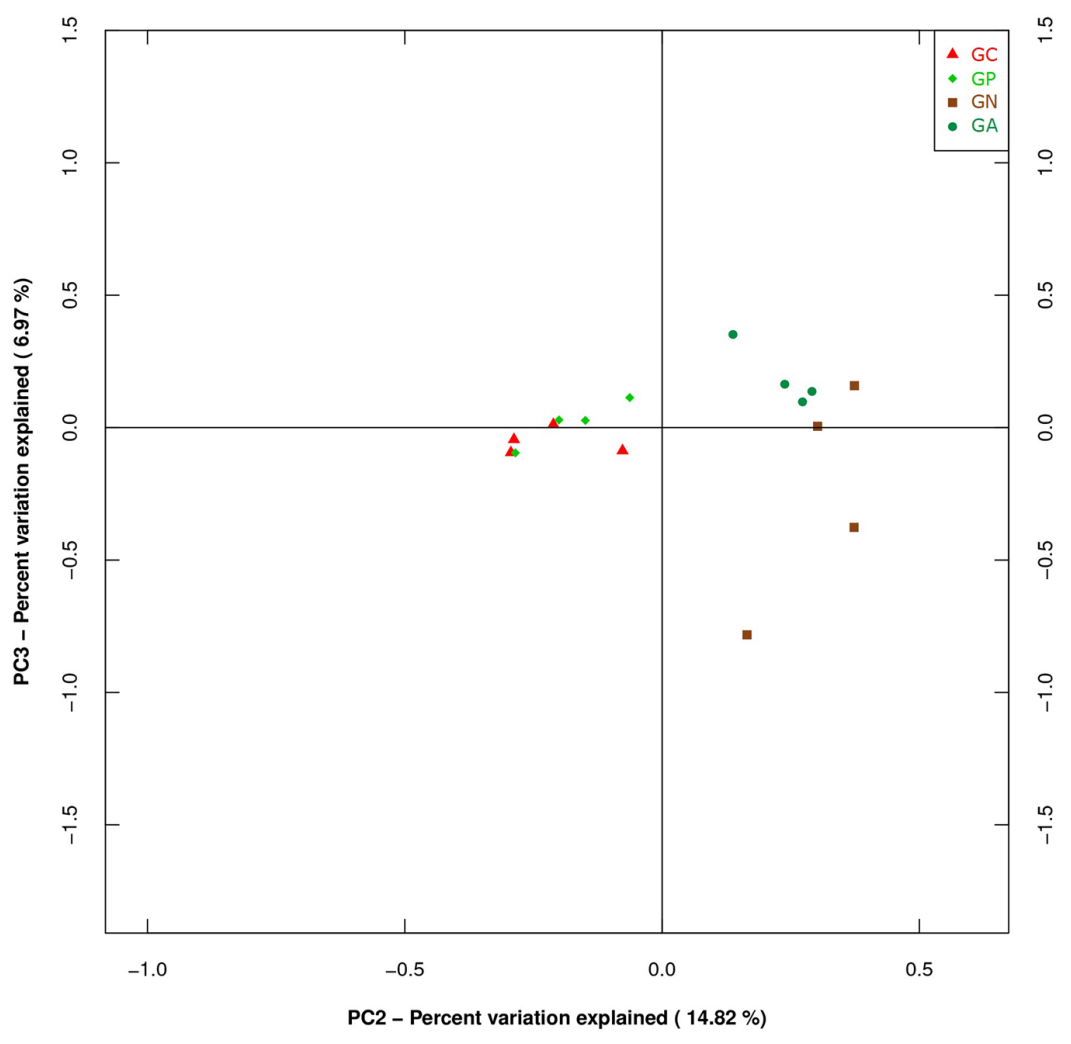

FIGURE 4 | Principal component analysis (PCA) plot based on the second and third PC of bacterial community taxonomic profiles from 16 mixed cecal samples in four groups. GC, control group; GP, phage-therapy group; GN, negative control group N; GA, antibiotic-therapy Group.

Studies using PCA for analyzing gut microbiota of rabbits receiving different treatments are very few. PCA is a method to covert a lot of observations into a set of linearly uncorrelated variables. Basically, the closer the dots on the PCA plot are, the more homologous they are. In our study, samples from Groups C and P had PCA plot points that appeared in a cluster. We assumed that the narrow antibacterial spectrum of bacteriophage ZRP1 preserved the existing microbiome. On the contrary, ciprofloxacin lactate, a broad-spectrum third-generation fluoroquinolone, might be harmful to YS2 (in the phylum Cyanobacteria) because ciprofloxacin is toxic to Cyanobacteria (Ebert et al., 2011). Antibiotics are widely used in animal disease treatment and prevention or feed efficiency improvement, but they destroy pathogens and commensal bacteria simultaneously. A short-term antibiotic treatment can shift the population structure of the microbiota and alter bacterial physiology, such as reduction of the amount and diversity of microbes, losses in the function of metabolism and the modulation of the immune system (Ferrer et al., 2017). Therefore, antimicrobial agents with minimal side-effects on commensal bacteria are badly needed (Toutain et al., 2016).

Normally, each individual phage has a very narrow host range that will benefit microbial community by affecting only a relatively small subset of bacteria (Dalmasso et al., 2014). The advantage of phage therapy is to specifically target pathogens while not to perturb the normal microbiota. If the normal gut microbiota is not disrupted in a designed phage therapy, they will provide protection to the gut from disease by stimulating the recovery of the immune response to pathogens (Belizario and Napolitano, 2015; McCarville et al., 2016).

Up to now, little was revealed about the role of phage therapy in shaping the gut microbiota and its possible influence on health. The potential impact of phages on bacterial population also relies on the opportunity of encounters between lytic phages and their host in vivo (Dalmasso et al., 2014). The result of our work suggested that the postulate that phage therapy has little impact on the gut bacteria ecology is wellfounded.

\section{CONCLUSION}

Rabbits orally infected with pathogenic E. coli can be cured by a single specific phage therapy. The phage removed nearly all host cells in vivo and had few effects on other bacteria. However, comparing to those received antibiotic treatment; phage-therapy 
rabbits had a little higher residual ZR1 load in cecum contents at the end of the 3-day trial. It deserves further study using a longer period to see whether phage therapy is as effective as antibiotic treatment.

\section{AUTHOR CONTRIBUTIONS}

Conceived and designed the experiments: JZ, YL, HY, and GB. Performed the experiments: JZ, CX, and SH. Analyzed the data: JZ and YL. Contributed reagents/materials: CX. Contributed to the writing of the manuscript: JZ and YL. Revised the manuscript: GB.

\section{REFERENCES}

Abuladze, T., Li, M., Menetrez, M. Y., Dean, T., Senecal, A., and Sulakvelidze, A. (2008). Bacteriophages reduce experimental contamination of hard surfaces, tomato, spinach, broccoli, and ground beef by Escherichia coli O157:H7. Appl. Environ. Microbiol. 74, 6230-6238. doi: 10.1128/AEM.01465-08

Al-Mamun, A., Mily, A., Sarker, P., Tiash, S., Navarro, A., Akter, M., et al. (2013). Treatment with phenylbutyrate in a pre-clinical trial reduces diarrhea due to enteropathogenic Escherichia coli: link to cathelicidin induction. Microbes Infect. 15, 939-950. doi: 10.1016/j.micinf.2013.08.007

Bauerl, C., Collado, M. C., Zuniqa, M., Blas, E., and Perez Martinez, G. (2014). Changes in cecal microbiota and mucosal gene expression revealed new aspects of epizootic rabbit enteropathy. PLOS ONE 9:e105707. doi: 10.1371/journal. pone.0105707

Belizario, J. E., and Napolitano, M. (2015). Human microbiomes and their roles in dysbiosis, common diseases, and novel therapeutic approaches. Front. Microbiol. 6:1050. doi: 10.3389/fmicb.2015.01050

Bischoff, C., Luthy, J., Altwegg, M., and Baggi, F. (2005). Rapid detection of diarrheagenic E. coli by real-time PCR. J. Microbiol. Methods 61, 335-341. doi: 10.1016/j.mimet.2004.12.007

Brecchia, G., Cardinali, R., Mourvaki, E., Collodel, G., Moretti, E., Dal Bosco, A., et al. (2010). Short- and long-term effects of lipopolysaccharide-induced inflammation on rabbit sperm quality. Anim. Reprod. Sci. 118, 310-316. doi: 10.1016/j.anireprosci.2009.06.016

Brecchia, G., Menchetti, L., Cardinali, R., Castellini, C., Polisca, A., Zerani, M., et al. (2014). Effects of a bacterial lipopolysaccharide on the reproductive functions of rabbit does. Anim. Reprod. Sci. 147, 128-134. doi: 10.1016/j.anireprosci.2014. 04.009

Bull, J. J., Levin, B. R., DeRouin, T., Walker, N., and Bloch, C. A. (2002). Dynamics of success and failure in phage and antibiotic therapy in experimental infections. BMC Microbiol. 2:35. doi: 10.1186/1471-2180-2-35

Carter, C. D., Parks, A., Abuladze, T., Li, M., Woolston, J., Magnone, J., et al. (2012). Bacteriophage cocktail significantly reduces Escherichia coli O157:H7 contamination of lettuce and beef, but does not protect against recontamination. Bacteriophage 2, 178-185. doi: 10.4161/bact.22825

Combes, S., Gidenne, T., Cauguil, L., Bouchez, O., and Fortun-Lamothe, L. (2014). Coprophagous behavior of rabbit pups affects implantation of cecal microbiota and health status. J. Anim. Sci. 2, 652-665. doi: 10.2527/jas.2013-6394

Dalmasso, M., Hill, C., and Ross, R. P. (2014). Exploiting gut bacteriophages for human health. Trends Microbiol. 22, 399-405. doi: 10.1016/j.tim.2014.02.010

Ebert, I., Bachmann, J., Kuhnen, U., Kuster, A., Kussatz, C., Maletzki, D., et al. (2011). Toxicity of the fluoroquinolone antibiotics enrofloxacin and ciprofloxacin to photoautotrophic aquatic organisms. Environ. Toxicol. Chem. 30, 2786-2792. doi: 10.1002/etc.678

Ferrer, M., Mendez-Garcia, C., Rojo, D., Barbas, C., and Moya, A. (2017). Antibiotic use and microbiome function. Biochem. Pharmacol. 134, 114-126. doi: 10.1016/j.bcp.2016.09.007

Gallois, M., Gidenne, T., Tasca, C., Caubet, C., Coudert, C., Milon, A., et al. (2007). Maternal milk contains antimicrobial factors that protect young rabbits

\section{ACKNOWLEDGMENT}

This work was supported by a grant from the National Rabbit Industry Technology System of China (CARS44-C-2).

\section{SUPPLEMENTARY MATERIAL}

The Supplementary Material for this article can be found online at: http://journal.frontiersin.org/article/10.3389/fmicb. 2017.00957/full\#supplementary-material

TABLE S1 | Total OTUs and tags of 16 mixed samples in four groups.

from enteropathogenic Escherichia coli infection. Clin. Vaccine Immunol. 14, 585-592. doi: 10.1128/CVI.00468-06

Hernandes, R. T., Elias, W. P., Vieira, M. A. M., and Gomes, T. A. T. (2009). An overview of atypical enteropathogenic Escherichia coli. FEMS Microbiol. Lett. 297, 137-149. doi: 10.1111/j.1574-6968.2009.01664.x

Hraiech, S., Bregeon, F., and Rolain, J. M. (2015). Bacteriophage-based therapy in cystic fibrosis-associated Pseudomonas aeruginosa infections: rationale and current status. Drug Des. Dev. Ther. 9, 3653-3663. doi: 10.2147/DDDT. S53123

Luz Maria Chacon, J., Lizeth Taylor, C., Carmen Valiente, A., Irene Alvarado, P., and Ximena Cortes, B. (2012). A DNA pooling based system to detect Escherichia coli virulence factors in fecal and wastewater samples. Braz. J. Microbiol. 43, 1319-1326. doi: 10.1590/S1517-83822012000400 0012

Mandal, S. M., Roy, A., Ghosh, A. K., Hazra, T. K., Basak, A., and Franco, O. L. (2014). Challenges and future prospects of antibiotic therapy: from peptides to phages utilization. Front. Pharmacol. 5:105. doi: 10.3389/fphar.2014. 00105

McCarville, J. L., Caminero, A., and Verdu, E. E. (2016). Novel perspectives on therapeutic modulation of the gut microbiota. Ther. Adv. Gastroenterol. 9, 580-593. doi: 10.1177/1756283X16637819

Milon, A., Oswald, E., and De Rycke, J. (1999). Rabbit EPEC: a model for the study of enteropathogenic Escherichia coli. Vet. Res. 30, 203-219.

Raya, R. R., Oot, R. A., Moore-Maley, B., Wieland, S., Callaway, T. R., Kutter, E. M., et al. (2011). Naturally resident and exogenously applied T4-like and T5-like bacteriophages can reduce Escherichia coli O157:H7 levels in sheep gut. Bacteriophage 1, 15-24. doi: 10.4161/bact.1.1.14175

Smith, H. W., and Huggins, M. B. (1982). Successful treatment of experimental Escherichia coli infections in mice using phage: its general superiority over antibiotics. J. Gen. Microbiol. 128, 307-318. doi: 10.1099/00221287-1282-307

Tanji, Y., Shimada, T., Fukudomi, H., Miyanaga, K., Nakai, Y., and Unno, H. (2005). Therapeutic use of phage cocktail for controlling Escherichia coli O157:H7 in gastrointestinal tract of mice. J. Biosci. Bioeng. 100, 280-287. doi: $10.1263 /$ jbb.100.280

Toutain, P. L., Ferran, A. A., Bousquet-Melou, A., Pelligand, L., and Lees, P. (2016). Veterinary medicine needs new green antimicrobial drugs. Front. Microbiol. 7:1196. doi: 10.3389/fmicb.2016.01196

Tsonos, J., Vandenheuvel, D., Briers, Y., De Greve, H., Hernalsteens, J. P., and Lavigne, R. (2014). Hurdles in bacteriophage therapy: deconstructing the parameters. Vet. Microbiol. 171, 460-469. doi: 10.1016/j.vetmic.2013. 11.001

Vasileiadis, S., Puglisi, E., Arena, M., Cappa, F., Cocconcelli, P. S., and Trevisan, M. (2012). Soil bacterial diversity screening using single $16 \mathrm{~S}$ rRNA gene $\mathrm{V}$ regions coupled with multi-million read generating sequencing technologies. PLoS ONE 7:e42671. doi: 10.1371/journal.pone.0042671

Wei, L., Huang, C., Yang, H., Li, M., Yang, J., Qiao, X., et al. (2015). A potent antiinflammatory peptide from the salivary glands of horsefly. Parasit. Vectors 8 , 556. doi: 10.1186/s13071-015-1149-y 
Xie, H., Zhuang, X., Kong, J., Ma, G., and Zhang, H. (2005). Bacteriophage Esc-A is an efficient therapy for Escherichia coli 3-1 caused diarrhea in chickens. J. Gen. Appl. Microbiol. 51, 159-163.

Zhang, J., Li, Z., Cao, Z., Wang, L., Li, X., Li, S., et al. (2015). Bacteriophages as antimicrobial agents against major pathogens in swine: a review. J. Anim. Sci. Biotechnol. 6, 39. doi: 10.1186/s40104-0150039-7

Zhao, J., He, L., Pan, L., Liu, Y., Yao, H., and Bao, G. (2017). Effect of a lytic bacteriophage on rabbits experimentally infected with pathogenic Escherichia coli. World Rabbit Sci. (in press).
Conflict of Interest Statement: The authors declare that the research was conducted in the absence of any commercial or financial relationships that could be construed as a potential conflict of interest.

Copyright $\odot 2017$ Zhao, Liu, Xiao, He, Yao and Bao. This is an open-access article distributed under the terms of the Creative Commons Attribution License (CC BY). The use, distribution or reproduction in other forums is permitted, provided the original author(s) or licensor are credited and that the original publication in this journal is cited, in accordance with accepted academic practice. No use, distribution or reproduction is permitted which does not comply with these terms. 